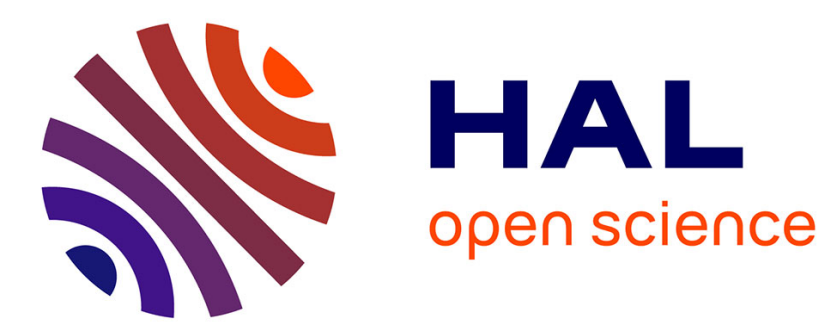

\title{
Guerre civile et œuvres pies en Algérie
}

Luis Martinez

\section{To cite this version:}

Luis Martinez. Guerre civile et œuvres pies en Algérie. Critique Internationale, 1999, 4, pp.127-137. 10.3406/criti.1999.1528. hal-01010826

\section{HAL Id: hal-01010826 \\ https: / hal-sciencespo.archives-ouvertes.fr/hal-01010826}

Submitted on 20 Jun 2014

HAL is a multi-disciplinary open access archive for the deposit and dissemination of scientific research documents, whether they are published or not. The documents may come from teaching and research institutions in France or abroad, or from public or private research centers.
L'archive ouverte pluridisciplinaire HAL, est destinée au dépôt et à la diffusion de documents scientifiques de niveau recherche, publiés ou non, émanant des établissements d'enseignement et de recherche français ou étrangers, des laboratoires publics ou privés.

\section{(이)(\$)}

Distributed under a Creative Commons Attribution - NonCommercial - NoDerivatives| 4.0 


\title{
Variations
}

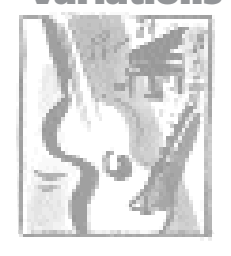

\section{Guerre civile et œuvres pies en Algérie}

\author{
par Luis Martinez
}

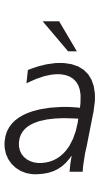

la fin des années quatre-vingt, nombre d'observateurs s'étonnaient des succès politiques du FIS : comment un parti qui prônait l'interdiction de la démocratie, du travail des femmes, de la musique et des paraboles pouvait-il mobiliser une majorité de votants ? Quelques années plus tard, on s'interrogeait de même sur les raisons pour lesquelles émirs de la guérilla et miliciens bénéficiaient de la sympathie d'une partie de la population. L'explication tient notamment à l'action sociale de ces groupes et de ces individus. Considérés comme extrémistes, violents, voire sanguinaires, ils n'en sont pas moins engagés dans des «stratégies de la bienfaisance », que Christophe Jaffrelot définit comme une « aide sociale apportée à des populations rencontrant des difficultés matérielles ou morales par des mouvements à l'idéologie affirmée $»^{1}$.

Loin d'être l'apanage d'un mouvement politique particulier (nationaliste, islamiste, régionaliste), ces stratégies caractérisent plutôt, en Algérie, des figures emblématiques incarnant la sainteté ou la force : celles du notable de l'État-FLN,

1. Christophe Jaffrelot, « CEuvres pies et rationalité économique en Inde », dans J.-F. Bayart (dir.), La réinvention du capitalisme, Paris, Karthala, 1994, p. 145. 
de l'élu du FIS, de l'émir de la guérilla et du milicien illustrent, chacune à sa manière, le type du bienfaiteur. Leur émergence est partiellement l'effet des défaillances de l'État. Car celui-ci est, dans l'imaginaire politique algérien, le bienfaiteur par excellence, les attentes qu'il suscite étant à la mesure des sacrifices autrefois consentis pour l'amener à l'existence. Comme l'écrit Abderrahman Moussaoui, « la population a fini par concevoir son rapport à l'État à travers un contrat d'échange tacite. En "donnant” à l'État son pétrole, qu'elle estime avoir chèrement payé au prix du sang de ses martyrs, elle en attendait une prise en charge totale, sans aucune autre contrepartie. L'État pouvait disposer librement du pétrole, à condition d'assurer en retour un contre-don, par la redistribution de revenus en salaires politiques et autres gratifications $»^{2}$. L'incapacité de l'État à assumer sa « générosité nécessaire »3 s'est traduite par l'émergence de figures de la bienfaisance dont certaines, paradoxalement, tirent précisément leurs ressources de leurs relations avec l'État.

Au surplus, la guerre, par les tragédies qu'elle suscite, constitue une opportunité historique de générosité et d'aide sociale ; surtout, elle multiplie les occasions d'enrichissement, donc aussi de largesses. Georges Duby relève que, dans l'Europe médiévale, la guerre, « activité économique régulière, d'importance considérable », remplissait aussi une fonction de redistribution sociale : «Quant aux princes, leur prestige était fonction de leur générosité : ils ne ravissaient - avec une cupidité qui paraît insatiable - que pour donner plus largement $\gg^{4}$. En Algérie, le détournement des fonds publics destinés aux familles de victimes des massacres par les notables locaux permet à ces derniers, en les redistribuant, d'accroître leur prestige. Il en est de même de l'aide apportée par les émirs de quartier ou les chefs de milices à des populations confrontées à l'insécurité économique et physique.

\section{Du clientélisme des notables de l'État-FLN à la bienfaisance des élus du FIS}

La pratique politique des notables de l'État-FLN constitue un des éléments explicatifs de la stabilité de l'État entre 1965 et 1988. Au moment de l'indépendance, le risque de guerre civile, après la meurtrière guerre de décolonisation, était patent. Les luttes de clans qui avaient opposé les maquisards durant cette dernière cessèrent avec la prise du pouvoir par Houari Boumediène ${ }^{5}$. La transformation des moudjahidin (combattants de la foi) en notables de la République socialiste permit, à la faveur d'une pratique politique clientéliste, de canaliser les tensions nées au cours de la guerre. Mohamed Harbi montre comment l'usurpation du pouvoir par la violence et le dépeçage du territoire par les maquisards sous la présidence Boumediène sanctionnent le règne des bandits politiques. Ceux-ci sont employés à pacifier la nation en organisant, au niveau local, un contrôle social et une redistribution économique comparables à l'action des caïds (fonctionnaires indigènes) 
et des bachaga de la période coloniale. La pratique politique des notables de l'ÉtatFLN repose non sur une stratégie de la bienfaisance mais sur la « prédation » comme facteur de « régulation politique $»^{6}$. Imbus d'un sentiment de supériorité qu'ils justifient par leur participation à la lutte pour l'indépendance, ils s'octroient le contrôle des richesses de la nation.

Dans un premier temps, l'accaparement des « biens vacants » (2 000 entreprises industrielles et commerciales, 200000 logements, 20000 mètres carrés de bureaux, etc.) permet de doter les nouvelles élites de l'Algérie indépendante des attributs de la puissance économique. Entre 1967 et 1968, les biens habous, propriétés incessibles et inaliénables gérées par le ministère des Affaires religieuses (lots de terre agricole, palmeraies, bains maures, terrains constructibles), sont nationalisés ; et la manne pétrolière née de la nationalisation des hydrocarbures permet l'instauration d'un État rentier redistributeur et garant de la paix sociale. Mais, à la fin des années quatre-vingt, le « charisme de guerre », atout des notables, s'épuise et la «domination charismatique » devient l'attribut des dirigeants du FIS : «Quand Abassi Madani est venu à Mazouna et à Relizane, les gens mettaient l'eau qui avait servi aux ablutions du Cheikh dans des flacons de pénicilline pour la garder en souvenir, pour la baraka $\gg^{7}$.

\section{La stratégie de bienfaisance des élus du FIS}

Contrairement aux notables de l'État-FLN, les élus municipaux du FIS ne disposent pas de la légitimité acquise par le don de soi à la patrie. Ils vont compenser ce handicap par un travail social en profondeur, d'autant plus nécessaire que leurs ennemis donnent d'eux une image détestable : « En Algérie, la peur de l'islam est un phénomène récent et insolite venu faire irruption dans notre imaginaire collectif, s'insinuer dans notre corps social. L'apparente source de cette angoisse collective est le mythique $\mathrm{Ghoul}^{8}$, décidément ressuscité, camouflé cette fois en islamiste alias intégriste alias fondamentaliste alias fanatique alias fasciste. L'image de ce médiéval Frère Ghoul qui hante une certaine Algérie affolée est un hideux barbu, inculte, fruste et intolérant, qui menace la paix sociale et violente les femmes $\gg$.

\footnotetext{
2. Abderrahman Moussaoui, « De la violence au djihâd », Annales HSS, nº 6, nov.-déc. 1994, p. 1330.

3. Georges Duby, Guerriers et paysans, VII'-XIIe siècles, premier essor de l'économie européenne, Paris, Gallimard, 1973, p. 63.

4. George Duby, op. cit., p. 64.

5. J. Leca et J.-C. Vatin, L'Algérie politique, institutions et régime, Paris, Presses de la FNSP, 1975, p. 395.

6. M. Harbi, chapitre « Régulation politique et prédation », dans L'Algérie et son destin. Croyants ou citoyens ? Paris, Arcantère, 1992, p. 192.

7. El Watan, 21 janvier 1998.

8. Ghoul est un personnage maléfique dans la littérature maghrébine, il est représenté sous la figure d'un monstre. La presse officielle appelait « Frères Ghoul » les islamistes.

9. El Mounqid (journal du FIS), n 27, 1 er $^{\text {octobre } 1990 .}$
} 
Déjà l'efficacité de l'action du parti auprès des sinistrés du tremblement de terre de Tipasa (29 octobre 1989) avait donné un aperçu de sa capacité à venir en aide aux plus démunis. Le contrôle de la majorité des municipalités, à partir de juin 1990, va renforcer sa capacité de bienfaisance : on prend enfin en compte les problèmes matériels des populations (pénurie de logements, coupures d'eau et d'électricité, crise des transports...) ; on met en place des marchés de produits alimentaires et de livres scolaires à très bas prix pour les plus pauvres. Cette politique accrédite la croyance populaire qu'en définitive le « parti de l'islam » était bel et bien la «solution ». À ces bienfaits matériels s'ajoute la certitude que des signes de Dieu sont apparus dans le ciel d'Alger sous la forme du « nom d'Allah clairement formé par des nuages qui se sont estompés peu à peu pour se dissoudre totalement dans l'air ». Cet événement attribue au FIS une mission grandiose : «Nous y croyons fermement, comme nous croyons fermement en l'avenir d'une République algérienne islamique prospère et heureuse. Dieu a voulu manifester ainsi sa toute-puissance pour renforcer les croyants dans leur conviction que ce qu'ils sont en train d'entreprendre est juste ${ }^{10}$. Les élus municipaux du FIS, en vue d'instaurer la république islamique sur ce qu'ils considèrent comme des « espaces libérés » de la terre d'Algérie, collectent et redistribuent aux pauvres la zakat (aumône) par le biais de « bureaux exécutifs du Trésor public de l'État musulman » créés dans leurs municipalités, ainsi que des lots de terrain. L'enthousiasme suscité par cette pratique est tel que le gouvernement retire aux municipalités (APC) leurs compétences en matière de distribution de terres pour les transférer aux agences foncières, dirigées par l'administration de l'État. Les municipalités, selon l'organe de presse du FIS, « ont également aidé des milliers de nécessiteux à construire leur propre habitation. Il est aussi intéressant d'évoquer l'expérience réussie des "marchés islamiques" organisés par le FIS pour améliorer le pouvoir d'achat des plus démunis aux occasions de grande consommation [mariages, Ramadan...]. Sans l'expérience réussie de la gestion des APC, le FIS n'aurait jamais pu obtenir le grand succès qui a été le sien aux législatives de $1991 »^{11}$. On ne saurait mieux dire que l'efficacité de son travail social et le « sérieux » de ses militants ${ }^{12}$ ont renforcé le crédit politique du parti et assuré sa victoire.

\section{Instaurer la « citadelle de l'islam »}

Investis d'une mission historico-religieuse, les élus municipaux du FIS bénéficient des attributs de la sainteté aux yeux de leurs électeurs. L'ambition d'instaurer le règne de la vertu dans les relations sociales et politiques implique de renverser l'ordre FLN, marqué du stigmate de la corruption ${ }^{13}$. Les élus du FIS ambitionnent aussi de mettre en place un ordre « sérieux » susceptible de pallier les dérives d'un État négligent : « Le sérieux est l'une des caractéristiques essentielles de l'engagement isla- 
mique actif », affirme un éditorial du FIS. Le devoir de bienfaisance, qui s'inscrit dans la perspective de bâtir « la citadelle de l'islam », doit s'exécuter par le biais d'une bonne gestion municipale. Le FIS est le premier parti à faire un usage intensif de l'informatique. On enregistre sur ordinateur les bénéficiaires des différentes donations, de même que les noms de ceux qui versent la zakat. Fiers de leur sérieux, les élus s'indignent des obstacles qu'on leur oppose : « Mais pourquoi ces égarés s'acharnent-ils contre l'islam ? Ils savent très bien qu'avec l'islam il sera mis fin à la corruption, au népotisme, aux passe-droit, aux vols, à l'injustice, à la hogra, à la dépravation, au gaspillage, aux négligences, à l'usure... Avec l'islam on créera la cité idéale débarrassée des hypocrites et des lâches [...]. La cité idéale sera celle où les gens vivront en frères en s'entraidant dans le bonheur et dans la peine. La cité idéale sera celle où l'aumône et la $z a k a t$ seront dispensées largement et sans réticences $\gg^{14}$. L'un des plus gros obstacles à de tels projets est le manque de ressources; les élus du FIS ne cessent de critiquer la volonté du régime de tarir les ressources financières du parti en vue de l'empêcher d'aider les plus démunis ${ }^{15}$. Le régime, dit Ali Belhadj, est prêt à accepter un parti islamique à la condition « que l'islam soit culte, prière, jeûne, récitation et culture, soit, mais quant à se mêler d'économie... »16.

Le prestige des élus du FIS se nourrit de la fin des largesses des notables du FLN. Ces derniers ne sont plus à même de contrôler le social, leur efficacité s'est amoindrie au cours de la décennie quatre-vingt. Le FIS ambitionne de reprendre à son compte la politique patrimonialiste de l'État-FLN, non d'y mettre un terme ${ }^{17}$. Aussi voit-il une grave menace dans le multipartisme qui émerge après les émeutes d'octobre 1988 : «La multiplication pathologique des partis gagne du terrain; à cette allure, le peuple algérien s'achemine vers une instabilité politique durable ${ }^{18}$. Bref, la démocratie risque d'empêcher la formation d'une variante islamiste de l'État-FLN. Le plus grand danger provient donc du soutien de la communauté internationale à ce régime, supposé avoir accepté une évolution démocratique.

10. El Mounqid, $\mathrm{n}^{\circ} 22$, mars 1990 .

11. El Mounqid, $\mathrm{n}^{\circ}$ 6, 14 décembre 1991.

12. Ignace Leverrier, « Le Front islamique du salut entre la hâte et la patience », dans G. Kepel (dir.), Les politiques de Dieu, Paris, Le Seuil, 1993.

13. Omar Carlier, « De l'islahisme à l'islamisme : la thérapie politico-religieuse du FIS », Cabiers d'études africaines, vol. XXXII (2), nº 126, 1993, pp. 185-219.

14. El Mounqid, n 40, 2 janvier 1991.

15. Le FIS a néanmoins bénéficié, dès sa reconnaissance officielle par le gouvernement algérien, des dons de l'Arabie saoudite. Le sultan Ibn Abdelaziz, dans une déclaration publiée par Al Charq al Awsat du 28 mars 1991, révèle que son pays a financé des mouvements islamistes en Algérie et en Tunisie.

16. «Lettre d'Ali Belhadj, prisonnier injustement, à Zéroual », 8 novembre 1994.

17. Lahouari Addi, « Néo-patrimonialisme et économie en Algérie », dans M. Camau (dir.), Changements politiques au Maghreb, Paris, CNRS, 1991.

18. El Mounqid, n $^{\circ}$ 30, 25 octobre 1990. 


\section{Crimes et bienfaisance : la stratégie des émirs}

La stratégie de bienfaisance des élus du FIS s'est donc articulée autour d'un travail social en profondeur, accompli avec efficacité, et accompagné de la perspective d'un avenir meilleur qu'apporterait l'instauration d'un État islamique. Après la dissolution du parti, en mars 1992, c'est la guerre civile, qui voit émerger une nouvelle figure de la bienfaisance, celle des « émirs ». Ceux-ci se donnent pour mission de « libérer » l'Algérie des « renégats » qui la gouvernent. Se définissant comme moudjahidin, ils offrent leur vie en sacrifice à la réalisation de cette œuvre pie, comme le proclame avec éclat l'Instance exécutive du FIS à l'étranger : «Vous qui avez donné vos personnes à Dieu, qui soulevez l'étendard de l'islam ensanglanté par le sacrifice des martyrs parmi vous, le conduisant au grand secours et à la victoire éclatante - que Dieu la permette - au moment où de nombreux musulmans retiennent leur sang et leurs biens par avarice. Vous, qui êtes libérés des séductions et des désirs de la vie, qui êtes contentés de la vie dure dans le sentier de Dieu, désirant seulement l'agrément et la satisfaction d'Allah, "réjouissez-vous du troc que vous avez troqué ! Voilà l'énorme succès !" (Coran IX, 111)»19.

Les émirs de la guérilla ont un mode d'accumulation de ressources qui repose sur l'usage de la violence. Pourtant, ils n'atteindront pas le même niveau de prestige que celui de cette figure emblématique de la réussite sociale que constitue, depuis les années soixante, l'entrepreneur militaire, ancien soldat de l'Armée de libération nationale reconverti en homme d'affaires. Dans un premier temps, la transformation de zones urbaines puis rurales en champs de guérilla leur permet bien d'étendre leur contrôle sur les ressources économiques. Mais, sous le coup de l'intrusion de nouveaux protagonistes - bandits, miliciens et tueurs des « escadrons de la mort »- leur autorité s'effritera, et avec elle le prestige qui entourait ces nouveaux $\ll$ lions de la montagne $»^{20}$.

La première génération des émirs a été marquée par le travail social du FIS : parallèlement aux activités de guérilla, les groupes armés s'efforcent de fournir des services à une population que la guerre entraîne dans la précarité et pire encore. Ils légitiment leur combat en invoquant l'impératif historico-religieux d'un État islamique et en redistribuant les bénéfices matériels du djihad. Ils viennent plus particulièrement en aide aux familles victimes de la répression militaire. Celle-ci consiste souvent, en effet, à détruire les maisons soupçonnées d'abriter des « terroristes ». Dès 1993, des familles n'ont ainsi même plus de toit. Elles sont alors, notamment dans le Grand Alger, prises en charge par les émirs qui les relogent dans les nombreux appartements abandonnés par leurs habitants en raison de l'insécurité croissante. Les émirs s'occupent également des familles de la plaine de la Mitidja qui fuient leurs hameaux pour se réfugier dans les petites communes de la ceinture d'Alger. Ces familles, malgré leur situation précaire, ne peuvent prétendre 
à l'aide publique, strictement réservée aux victimes des groupes islamistes, puisqu'elles fuient une région qui subit au contraire la violence de l'État (bombardement par l'armée des maquis de l'Atlas blidéen) ${ }^{21}$.

Mais les groupes armés islamistes commencent à être évincés du Grand Alger à partir de 1996, et les populations qu'ils « protégeaient » sont désormais aux prises avec des bandes proprement criminelles. La déstructuration des familles sous l'effet de ces multiples chocs (massacres, viols, déracinement, insécurité) se traduit par le développement de la prostitution et de la mendicité.

Entre 1992 et 1995, les émirs du GIA (Groupement islamique armé) et de l'AIS (Armée islamique du salut, organisation militaire du FIS) sont parvenus à asseoir leur autorité principalement grâce à leur action sociale. Dans les communes de la banlieue Sud et les villages de la Mitidja, ils remplissent une fonction de redistribution économique essentielle. L'arrêt du commerce informel (trabendo), la destruction des entreprises publiques et la fuite des responsables de petites entreprises privées plongent la population dans l'insécurité économique. À la faveur des bénéfices accumulés dans le djihad (dons de fidèles acquis à la cause d'un État islamique, attaques de banques, racket, etc.), les émirs créent des liens de clientèle avec des individus confrontés à des difficultés sociales et économiques, qu'ils rétribuent pour divers services. La raréfaction des ressources renforce leur pouvoir de redistribution. Ces années constituent l'unique moment de la guerre où la guérilla est en osmose avec son environnement humain. Accusés des pires crimes par le régime, les émirs n'en demeurent pas moins « comme poissons dans l'eau » au sein des communes naguère gérées par le FIS, où la version officielle des événements est considérée comme pure propagande. Leurs largesses apparaissent à la population qui en bénéficie comme un gage de leur sincérité et de leur dévouement à la cause qui les anime. En 1994, Ali Belhadj, la figure emblématique du FIS, écrit de sa prison : « Ces jeunes qui ont pris les armes aujourd'hui auraient pu être les fidèles serviteurs de la religion et de l'État, si celui-ci avait suivi la voie pacifique. Je les connais, car j'ai été élevé parmi eux, et je connais leur désintéressement, leur amour de la religion. Ils ne demandent ni poste ni honneurs $»^{22}$. Ainsi, pour le prêcheur le plus populaire, les émirs demeurent, en dépit de toutes les accusations dont ils sont l'objet, des bienfaiteurs qui ont fait don de soi.

19. « Lettre aux moudjahidin », dans Mots de Vérité, document du FIS, avril 1995.

20. Abdelkader Djeghloul montre comment se chevauchent en Algérie le personnage du « résistant » et celui du « hors-laloi », qualifié dans la poésie de « chevalier des crêtes » : «Le hors-la-loi, c'est d'abord celui qui a osé se lever et mourir, celui qui a relevé l'affront fait à la communauté. Il est l'anti-État, mais aussi le contre-État, le "sultan", le "lion" ». Éléments d'histoire culturelle algérienne, Alger, Enal, 1984, p. 185.

21. Si, à partir de 1997, d'autres familles, cette fois cibles des groupes islamistes, fuient à leur tour des villages de la Mitidja, elles ne bénéficient pas davantage d'aides financières publiques, cette fois en raison de leur impossibilité de fournir des attestations justifiant leur domicile : beaucoup de maisons ont été construites sans autorisation administrative.

22. « Lettre d'Ali Belhadj,...», op. cit. 
Certes, la routinisation du conflit entame peu à peu ces représentations positives des émirs, plus particulièrement ceux du GIA depuis les massacres perpétrés contre les civils. Mais surtout, la lutte antiguérilla que met en place le régime sous la houlette du général Mohammed Lamari à partir de 1993 les prive progressivement de ressources. En outre, la liquidation physique de leur première génération amène des successeurs dépourvus du prestige qui entourait ceux qui avaient côtoyé les élus du FIS : entre 1993 et 1995, tous ceux qui, par conviction religieuse et politique, avaient pris les armes mus par un profond sentiment d'injustice après l'interruption du processus électoral sont éliminés. À partir de 1995, s'autoproclament émirs des jeunes dont le pouvoir repose sur la terreur. Produits de la guerre, ils sont à la marge du monde du crime. Sans véritable stratégie politique, ils font de la violence un mode de vie. L'électorat populaire du FIS cesse (autant que faire se peut...) de financer ces nouveaux combattants en qui il voit des bandits opportunément convertis à l'islamisme. Les quartiers du Grand Alger se transforment en ghettos où s'entretuent à huis clos des repris de justice en lutte pour le monopole du racket. Cette dérive vers le banditisme fait craindre une délégitimation de toute la guérilla. C'est pourquoi les émirs de l'AIS s'évertuent à souligner les différences manifestes entre leurs actions et celles de ces «parvenus ». Afin de se disculper de toute responsabilité, ils diffusent, au lendemain des massacres de Bentalha, de Raïs et de Beni Messous (1997), des cassettes vidéo qui montrent comment les « vrais moudjahidin » tentent, tant bien que mal, de venir en aide aux familles martyrisées. Et pourtant, les revenus manquent aux compagnies de l'AIS : celle-ci, en s'interdisant le racket généralisé de la population sur le modèle du GIA, ne parvient plus à subvenir à ses besoins. Plus d'un « repenti » a raconté l'état de dénuement dans lequel se trouvent les moudjahidin dans les massifs de l'Ouarsenis en 19961997. La lutte antiguérilla a réduit les combattants de l'AIS à survivre dans la montagne, dépendant, pour se nourrir, des dons des paysans. Mais l'aide aux familles meurtries par les raids du GIA montre que les émirs de l'AIS inscrivent toujours leur action politique dans le registre du sacrifice à la communauté et cherchent à démontrer, face à leurs détracteurs, que leurs actions sont dictées par une éthique. Toutefois, littéralement épuisés par la stratégie de l'armée, ils sont contraints d'accepter une trêve unilatérale et sans condition en octobre 199723. Leur bienfaisance qui, au début du conflit, leur assurait une légitimité, se trouve, avec l'émergence des milices, progressivement remise en cause.

23. Dans un communiqué de février 1998, l'AIS déclare que « la trêve, amorcée depuis plus d'une année et ayant reçu bonne acceptation de la part de l'ANP [l'armée], est considérée comme une bonne œuvre et une position patriotique audacieuse qui a contribué d'une manière appréciable à la diminution des tueries ». Et elle précise que trente groupes armés autonomes ont rejoint le « camp de la trêve ». 


\section{Réinvention du notable : miliciens et politiques}

À partir de 1996, le gouvernement dispose d'une réserve en dollars qui ne cesse de croître, pour atteindre les 8 milliards en 1998. En effet, le régime, qui était en cessation de paiement en 1993 (les revenus issus des exportations avoisinent cette annéelà le montant du remboursement de la dette), s'était alors lancé, sous la houlette du FMI, dans un plan d'ajustement structurel accompagné du rééchelonnement de sa dette. Les revenus dégagés par les réformes économiques sont investis, dans un premier temps, dans la politique sécuritaire. Le plan d'ajustement structurel permet donc non seulement le rétablissement macro-économique mais aussi la mise sur pied d'un redoutable appareil de guerre composé de 200000 miliciens, 80000 gendarmes, 50000 gardes communaux et 80000 soldats intégrés dans un corps d'armée spécialisé dans la lutte antiguérilla. La dynamique de la guerre a créé de nouveaux notables : les chefs de groupes d'autodéfense, qui se font une place au soleil et apparaissent comme de véritables seigneurs de la guerre. Leur allégeance envers le pouvoir central demeure lâche, bien qu'ils soient financés par le gouvernement. Nombre d'entre eux disposent d'une richesse personnelle qui les met à l'abri de toute dépendance. Ces miliciens deviennent vite incontournables. Ce sont eux qui sont à l'origine de la déstructuration des réseaux économiques de la guérilla. Leur quadrillage du territoire prive les groupes armés islamistes d'une de leurs principales sources de financement, l'« impôt révolutionnaire » exigé des civils au passage des « faux barrages ». La mise en place de « comités d'autodéfense » dans les villages réduit les razzias perpétrées par la guérilla. Vivant au cour des zones de combat, les chefs de groupes d'autodéfense retournent progressivement la population en leur faveur, dissuadent les commerçants de financer les émirs et offrent aux jeunes sans ressources une opportunité d'emploi en les recrutant dans leurs milices. En délogeant les groupes des petits villages, des hameaux et des domaines agricoles à l'abandon, ils « pacifient » le Piémont blidéen et quadrillent une grande partie de la Mitidja.

Regroupés autour de l'Association des patriotes de la Mitidja, dont l'inspirateur est Mohamed Sellami (« le père des patriotes »), les chefs de milices deviennent les nouveaux acteurs hégémoniques de l'espace local. Ils ambitionnent de restaurer l'ordre et la sécurité dans les villages et de leur « redonner la vie ». En septembre 1996, l'Association organise une « soirée du souvenir »; d'autres cérémonies suivront. On met en place des structures sportives. Les nouveaux bienfaiteurs encouragent l'organisation de noces dont ils prennent les frais à leur charge, invitent les habitants à danser et les cortèges à klaxonner. Mais leur générosité ne permet pas pour autant de faire oublier les jalons de leur ascension sociale. Certains sont sous le coup d'accusations de meurtre, de viol et de vol, d'autres siègent au Conseil de la nation depuis leur nomination par le président Liamine Zéroual. Quelques-uns cumulent accusations et nomination... 
Leur montée en puissance suscite des inquiétudes quant à la captation des ressources publiques. Certains chefs de groupes d'autodéfense s'approprient ainsi des terres en voie de privatisation et détournent des indemnités financières destinées aux familles victimes des massacres. Le pouvoir, afin de minimiser sa dépendance envers les chefs de milices, favorise alors un processus de « politisation » susceptible de produire de nouveaux intermédiaires. La création du RND (Rassemblement national démocratique) a ainsi pour but de remettre l'autorité locale à des élus politiques. La « victoire » (très contestée en raison du truquage généralisé du scrutin) de ce parti aux élections législatives et municipales de juin et octobre 1997 limite l'autorité des milices.

Ainsi, s'appuyant sur ses rentrées financières régulières, le gouvernement a produit de nouvelles notabilités chargées d'assurer la paix sociale et la reconstruction des communes détruites par les années de guerre : « Le wali-délégué de Baraki a fourni hier, au siège du gouvernorat du Grand Alger, le montant de la première étape de réhabilitation de vingt-quatre infrastructures détruites ou saccagées. Un montant qui s'élève à 189 millions de dinars, financé sur le budget de l'État, du gouvernorat et des APC de Baraki (43 millions), des Eucalyptus (64 millions) et de Sidi Moussa (82 millions) »24. L'injection de sommes aussi considérables dans les communes les plus dévastées par la violence a des effets divers. Si les accusations de détournement par les chefs de groupes d'autodéfense sont nombreuses, elles n'épargnent pas les élus locaux. Ces derniers se distinguent dans le paysage par la hauteur de leurs maisons : dans les villages, il était de coutume que les maisons des notables du FLN soient les plus hautes mais, depuis quelque temps, certains élus du RND édifient des maisons encore plus élevées. Contraints de vivre dans un environnement hostile au régime, les nouveaux notables bénéficient de la libéralisation du secteur de la sécurité : plus de soixante sociétés assurent à différents clients les services d'une protection privée. Né d'une décision administrative, sans véritable soutien populaire, le RND est l'un des partis politiques les plus riches. Dans l'esprit de ses promoteurs, il s'agissait de recréer, à travers une stratégie clientéliste, une base électorale susceptible de remplacer celle du FLN. À partir de 1997, dans les communes du Grand Alger où le FIS avait réalisé ses meilleurs scores électoraux, des cadeaux sont distribués à la population. Les plus jeunes ne sont pas oubliés : des baby-foot sont offerts aux bars de quartiers, ainsi que des salles de jeux. De même sont installées, dans certaines de ces communes, des cliniques privées, équipées de matériel en provenance de Thailande, qui mettent un terme à la longue période de désinvestissement social. Ces cliniques remplissent une fonction socio-politique considérable en raison de l'effondrement des soins publics et de la quasi-impossibilité d'effectuer ces soins en France du fait des restrictions à la délivrance des visas. La politique du RND dans ces communes s'effectue en coopération avec le parti islamiste modéré de Mahfoudh Nahnah, le MSP (Mouvement de la société pour la paix). 
Rien ne garantit un succès comparable à celui du FIS à cette opération de promotion de nouveaux entrepreneurs politiques sur le modèle des notables du FLN. Il y manque un ingrédient sans doute essentiel, la foi en une idéologie mobilisatrice.

La stratégie de bienfaisance du FIS a été l'un des facteurs de son succès. Son institutionnalisation dans le champ politique lui a permis de rationaliser cette pratique, comme l'a illustré sa gestion des municipalités. Mais le « rétrécissement » de l'État du fait de la guerre a suscité une concurrence nouvelle entre différents types de bienfaiteurs. Alors que, sous l'État-FLN, seuls les notables étaient en mesure de pratiquer une large générosité, la décennie quatre-vingt-dix a vu se briser le monopole de la bienfaisance. Après les élus du FIS, émirs de groupes armés, chefs de milices et « élus de l'administration » ont mis en place des stratégies de la bienfaisance qui n'ont rien à envier aux anciennes pratiques des notables du FLN. Il est vrai que les transformations économiques qui se sont opérées au cours de la décennie jouent aussi leur rôle dans la privatisation des œuvres sociales. La libéralisation de nombreux secteurs économiques s'est accompagnée de l'émergence de nouveaux «big men $»^{25}$ sur qui l'État se « décharge » de ses fonctions régaliennes ${ }^{26}$. L'émergence de ces nouveaux caïds s'accompagne d'une économie de prédation dont Georges Duby souligne qu'au Moyen-Âge elle a favorisé l'expansion du commerce, « qui ne fut en fait que la très progressive et toujours incomplète insertion d'une économie du pillage, du don et de la largesse dans les cadres de la circulation monétaire $\gg 27$.

Mais la prolifération de bienfaiteurs ne compense pas, pour la population, la mise à l'écart des élus du FIS, qui avaient su articuler leur travail social à un apport politico-moral. C'est partiellement en vain que leurs successeurs s'évertuent à « redonner la vie » en offrant des biens.

24. El Watan, 23 février 1998.

25. Jean-Francois Médard, « Le "big man” en Afrique : esquisse d'analyse du politicien entrepreneur », L'Année Sociologique, $\mathrm{n}^{\circ} 42,1992$, pp. 167-192.

26. Béatrice Hibou, « Retrait ou redéploiement de l'État ? », Critique internationale, n 1, automne 1998, p. 154.

27. Georges Duby, op. cit., p. 69. 